\title{
METHODS OF THE TOMOGRAPHIC VISUALIZATION OF COMPLICATED CYSTS OF THE NECK
}

\author{
Lalita Yunusova $^{1)}$, Toru Aoyama ${ }^{2)}$, Matlyuba Khalmatova ${ }^{3)}$, Dilorom Djakhangirova ${ }^{3)}$, \\ Shakhnoza Ortikbaeva ${ }^{3)}$, Sobirjon Mamarajabov ${ }^{3)}$, Junichi Sakamoto ${ }^{4)}$, Nigina Abduxalik-Zade ${ }^{3)}$ \\ ${ }^{1)}$ Tashkent State Dental Institute, Republic of Uzbekistan \\ ${ }^{2)}$ Department of Surgery, Yokohama City University, Japan \\ ${ }^{3)}$ Tashkent pediatric medical institute, Republic of Uzbekistan \\ 4) Tokai central hospital, Japan
}

\begin{abstract}
Background: The present study attempted to improve the multimodal diagnosis of complicated neck cysts.

Materials and methods: 121 patients diagnosed to have neck cysts were entered in this study. The studies were performed on SLE-501 devices (Lithuania), Affiniti-70 (Philips, Holland) with linear sensors at a frequency of 7.5 and $12 \mathrm{MHz}$. Spiral CT was performed on a Somatom Emotion 6 apparatus (Siemens, Germany) using standard axial sections.

Results: Significant differences between the groups with complicated cysts of the neck were found with regard to the size, homogeneity, and extracapsular spread. Local infiltration was found to have a greater long axis $(\mathrm{p}<0.001)$, short axis $(\mathrm{p}<$ $0.001)$, and height $(\mathrm{p}<0.001)$. They were less likely to have extracapsular spread $(\mathrm{p}=0.044)$ or septations $(\mathrm{p}=0.059)$ and more likely to be homogeneous $(\mathrm{p}<0.001)$.

Conclusion: The use of modern methods of complementary capabilities of computed tomography and magnetic resonance imaging in our study allowed us to evaluate in detail all of the features of complicated cysts of the neck, which in turn helped determine the most appropriate treatment strategy in each case.
\end{abstract}

Keywords: Complicated cysts, computer and magnetic resonance tomography, ultrasound, cystic lesions.

(Received October 28, 2020; Accepted November 16, 2020)

\section{Background}

Complicated cysts of the neck are a separate group of cystic lesions of the neck structures. Complications of cysts and cysts of the neck may differ, and their differential diagnosis is difficult. The most frequent complication is an infected cyst, which in most cases leads to complete and incomplete fistulas, abscesses neck, abscesses, low current pyo-inflammatory processes, such as local infiltration, lymphadenitis. Complication of side brush can branchiogenic cancer. Median cysts show characteristic abscess formation and local infiltration around the cystic formation.

The clinical evaluation of complicated cysts of the neck and their complications are not always satisfied maxillofacial surgeons; they need precise information concerning the nature of the complications, the location and boundaries, and the state of the surrounding compli-

Corresponding author: Toru Aoyama, Department of Surgery, Yokohama City University, 3-9 Fukuura Kanazawa-ku, Yokohama, Japan. E-mail: t-aoyama@lilac. plala.or.jp cated cyst neck structures.

\section{Materials and methods}

121 patients diagnosed to have neck cysts were entered in this study. Magnetic resonance imaging was the reference method for evaluating the diagnostic effectiveness of ultrasound, computed tomography (CT) in complicated neck cysts. The studies were performed on SLE501 devices (Lithuania), Affiniti-70 (Philips, Holland) with linear sensors at a frequency of 7.5 and $12 \mathrm{MHz}$. Spiral CT was performed on a Somatom Emotion 6 apparatus (Siemens, Germany) using standard axial sections. Patients were examined with a 1.5-T MRI system (Intera Achieva 1.5 T Pulsar; Philips Medical Systems, Best, Netherlands).

\section{Results}

The study was conducted in the clinic of the Tashkent State Dental Institute. Of 121 patients with neck cysts, purulent-inflammatory complications of cysts were de- 
tected in 15 patients. Of these, 9 have local infiltrates, 2 phlegmons and 4 abscesses. We detected local infiltrative changes in cystic neoplasms of the neck in nine patients. Infiltrative changes in cystic formations represented a zone of sizes from 30 to $50 \mathrm{~mm}$ in 7 patients $(77.7 \%)$ with uneven, clear, in 2 patients $(22.3 \%)$ sizes exceeding $70 \mathrm{~mm}$, with fuzzy contours and the absence of a capsule. Acoustic zones were hypoechoic in four and showed mixed echogenicity in three patients. Nonhomogeneous echostructures containing small fluid inclusions up to 10 $\mathrm{mm}$ in diameter were noted. A clear differentiation of the zone from the surrounding tissues due to edema of the subcutaneous fat was absent in 3 patients (33.4\%). When conducting color doppler mapping (CDM), the blood flow inside the cystic formations with infiltrative changes was not determined.

On an ultrasound study in B-mode with inflammatory changes in patients, we noted an increase in the size of the formation compared to its original size, thickening of the capsule, and the appearance of hyperechoic suspension. In the study of complicated cystic neck formations in two patients with a lateral cyst of the neck, phlegmon of the soft tissues of the neck was revealed, the cause of which was abscessed cysts against the background of di- abetes mellitus. According to ultrasound data in B-mode, against the background of a lack of differentiation of soft tissues into layers, phlegmon in turn was represented by an extensive zone located subfascial and intramuscularly, occupying more than two areas with fuzzy contours and the absence of a capsule.

The ultrasonic characteristics mainly constituted signs of mixed echogenicity and the presence of a fluid area, denoting a cyst. The surrounding muscle tissue was characterized by increased echogenicity with a diffuse change in the echostructure compared to the opposite side. In one patient, we noted signs of inflammation in the muscles in contact with the cystic cavity, while the fibrous layers appeared hypoechoic due to the presence of inflammatory exudate. With color duplex scanning (CDS), we did not detect any blood flow inside the cyst complicated by phlegmon. Patients also underwent CT and MRI, and the results of such radiation research techniques confirmed the data obtained during the ultrasound examination. When performing work, among the complicated cystic formations of the neck there were such complications as abscessing of the cyst. Depending on the localization, 2 patients with lateral cysts had abscess cysts (Figs. 1 and 2) and in 2 patients with median cysts.
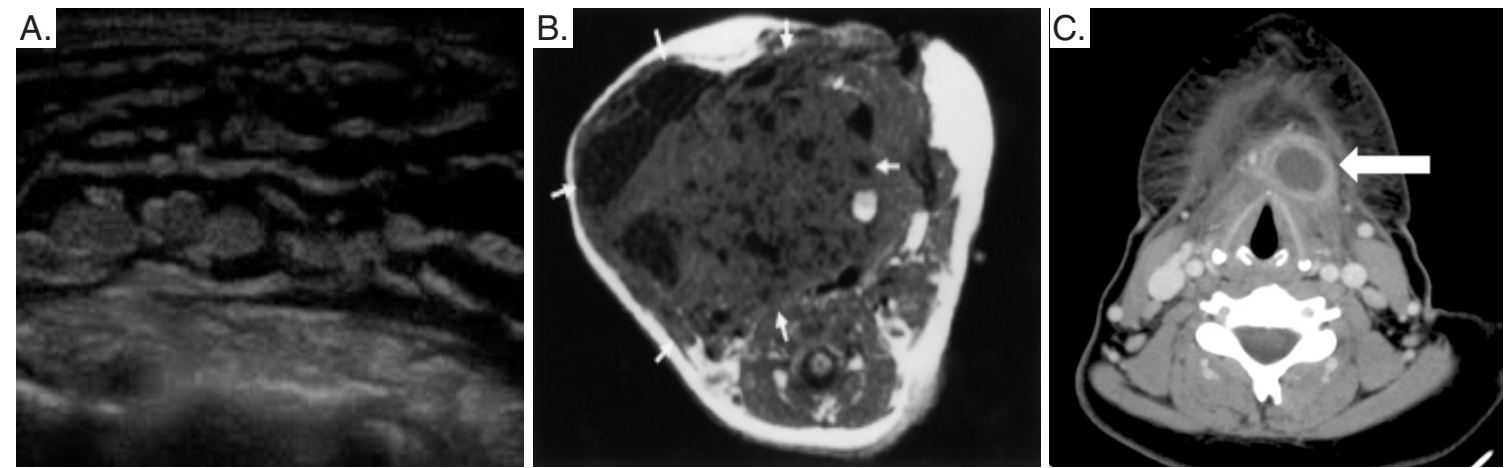

Fig. 1 A - Echogram and MRI chart of patient Sh., 39 years old, - axial plane and axial section with a lateral cyst of the neck complicated by phlegmon.

C - CT of patient S., 19 years old, on an axial section with a median cyst of the neck, complicated abscess formation and inflammatory changes in the surrounding fiber.

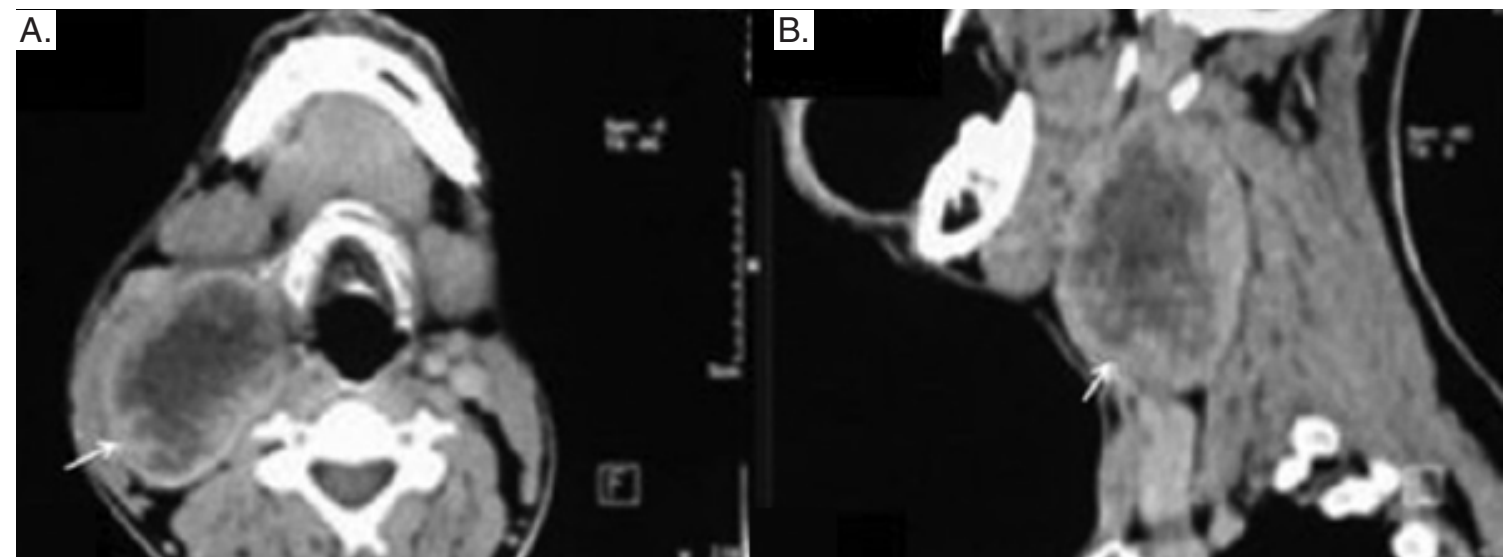

Fig. 2 CT of patient D., 42 years old. - axial and sagittal sections. Lateral cyst of the neck, complicated by abscess formation. 
An ultrasound study in B-mode conducted in patients revealed the presence of hypoechoic formations up to $30-40 \mathrm{~mm}$ in diameter with localization along the lateral surface and midline of the neck. In $5 \%$ of cases, the formation was of mixed echogenicity, and heterogeneous echostructure and echosuspension were detected in 3 cases. Rough, fuzzy contours were noted in two patients, and the presence of a capsule was noted in two patients. Blood flow in the structure of the cyst was not detected on CDS in any patient. To clarify the anatomical and topographic features of abscessed cysts and determine their relationship with the surrounding structures, MRI was performed. On MRI, lateral cysts of the neck were found to be located closely to the vascular bundle, while median cysts showed no relationship with the surrounding structures. Among 4 patients, 3 patients were diagnosed neck cysts complicated by an abscess using multislice computed tomography (MSCT). On CT, images appeared diffuse, without clear boundaries obliteration of the cellular spaces or single-, multi-chamber fluid accumulation in patients, and single sections of gas with edema of the surrounding tissues. In the present study, we observed cystic formations with reactive lymphadenopathy in 11 patients. We detected inflammatory processes localized in the deep cervical lymph nodes in 5 patients (45.5\%), in the submandibular lymph nodes in 3 patients $(27.3 \%)$, in the parotid lymph nodes in 2 patients $(18.2 \%)$, and in the submental areas in 1 patient. Furthermore, we observed lesions in the lymph nodes on the side of the cystic formation, but two patients were diagnosed with bilateral enlargement of the lymph nodes. Of note, in $50 \%$ of cases, the observed changes were of a multiple nature. The analysis of ultrasound signs obtained in B-mode and CDS data of edematous-infiltrative changes in the lymph nodes suggested that the size of the lymph nodes reached $30 \mathrm{~mm}$ in diameter, with fuzzy, uneven contours in 7 patients $(63.7 \%)$ while the remaining patients showed clear, uneven contours. The shape of the lymph nodes was round, with an increased blood flow found.

\section{Discussion}

Complicated cysts of the neck are mainly inflammatory. While these lesions may appear similar on CT and MRI, their characteristic locations-midline or lateral, deep or superficial-suggest a specific diagnosis. Devine et al. ${ }^{1)}$ conducted a retrospective review of patients with neck cysts who underwent neck dissection and found that $50 \%$ of the population showed complicated cysts of the neck. Haynes et al. ${ }^{2}$ reviewed a similar set of cystic squamous cell carcinoma (SCC) and found that most of the patients had origins of either. Kho et al. ${ }^{3)}$ considered the use of positron emission tomography (PET)/CT to provide more information regarding cystic masses in the neck but concluded that PET did not improve the diag- nostic accuracy and was often misleading. These studies, however, did not note any significant differences in homogeneity or the presence of septations between groups of complicated cysts of the neck. Rohof et al. ${ }^{4)}$ described the imaging characteristics regularly associated with complicated cysts of the neck with lymph nodes, such as central necrosis, nodal grouping, and nodule size. As described in the literature, central necrosis refers to an area of irregular hypodensity within the lymph node. Irregular hypodensity cystic masses within the lymph node. Pitner et al. ${ }^{5)}$ performed a multi-institutional study to determine the sensitivity and specificity of various sizes and radiographic criteria for detecting complicated cysts using MRI and CT. Those authors showed that CT performed better than MRI, with a $84 \%$ negative predictive value and $50 \%$ positive predictive value using a cysts wall size criterion of $1 \mathrm{~cm}$ or the presence of internal abnormality. Our results are comparable to those of that study, although the authors did note a greater significance for the presence or absence. In addition, complicated branchial cleft cysts tended to be larger would correlate with the low positive predictive value associated with size criteria. More research comparing the radiographic features of complicated cysts masses with a larger sample size and in a prospective fashion should be conducted to better illustrate the sensitivity and specificity of using different radiographic criteria. A retrospective study using MRI criteria would be helpful for differentiating complicated cysts masses of the neck from malignant lymph nodes by assessing the difference in the signal on T2-weighted imaging, the definition of margins on unenhanced imaging, and cellularity on diffusion-weighted imaging. The present study revealed that $31 \%$ of patients had cystic adenopathy without extracapsular spread or septations, while $38 \%$ of patients with complicated cysts had aggressive features mimicking phlegmon and abscesses with either septations or cyst capsule spread. With this degree of overlap, a high level of suspicion regarding cystic neck masses is required. In some of these patients, the use of clinical information, such as the patient age and disease process, may provide more information to aid the diagnosis.

\section{Conclusion}

In conclusion, modern CT and MRI modalities have no alternative in the diagnosis of neck cysts and their complications, since these methods of radiation diagnostics can accurately assess the nature of the complication, especially in the initial clinical picture. Using the modern capabilities of complementary methods of CT and MRI in our studies allowed us to evaluate in detail all of the signs of complicated neck cysts, which in turn determined the choice of an adequate treatment tactic in each case. 


\section{Acknowledgments:}

This study is supported, in part, by the nonprofit organization Epidemiological and Clinical Research Information Network (ECRIN).

\section{References}

1) Devine CM, Park E, Vachhani N, Butler R, Krakovitz P. Preoperative ultrasound for the diagnosis of thyroglossal duct cysts: A validation study. Int J Pediatr Otorhinolaryngol. 2019;122:89-92. Epub 2019 Mar 29. PMID: 30991206. doi: 10.1016/j.ijporl.2019.03.029

2) Haynes J, Arnold KR, Aguirre-Oskins C, Chandra S. Evaluation of neck masses in adults. Am Fam Physician. 2015;91(10):698-706. PMID: 25978199
3) Kho JPY, Prepageran N. Huge brachial plexus schwannoma, masking as a cystic neck mass. AME Case Rep. 2018;2:41. PMID: 30363802; PMCID: PMC6182036. doi: 10.21037/acr.2018.08.01

4) Rohof D, Honings J, Theunisse HJ, Schutte HW, van den Hoogen FJ, van den Broek GB, Takes RP, Wijnen MH, Marres HA Recurrences after thyroglossal duct cyst surgery: Results in 207 consecutive cases and review of the literature. Head Neck. 2015;37(12):1699-704. Epub 2014 Sep 25. PMID: 24985922. doi: 10.1002/hed.23817

5) Pitner H, Elmaraghy C, Fischer B, Onwuka A, Rabe A, Walz P. Diagnostic Accuracy of Midline Pediatric Neck Masses. Otolaryngol Head Neck Surg. 2019;160(6):1111-1117. Epub 2019 Feb 5. PMID: 30717638. doi: 10.1177/0194599819827845 